\title{
Structure of aluminum atomic chains
}

\author{
Prasenjit Sen, ${ }^{1}$ S. Ciraci, ${ }^{1,2}$ A. Buldum, ${ }^{3}$ and Inder P. Batra ${ }^{1}$ \\ ${ }^{1}$ Department of Physics, University of Illinois at Chicago, Chicago Illinois 60607-7059 \\ ${ }^{2}$ Department of Physics, Bilkent University, Bilkent, Ankara 06533, Turkey \\ ${ }^{3}$ Department of Physics and Astronomy, The University of North Carolina at Chapel Hill, Chapel Hill, North Carolina 27599
}

(Received 11 April 2001; revised manuscript received 20 June 2001; published 30 October 2001)

\begin{abstract}
First-principles density-functional calculations reveal that aluminum can form planar chains in zigzag and ladder structures. The most stable one has equilateral triangular geometry with four nearest neighbors; the other stable zigzag structure has wide bond angle and allows for two nearest neighbors. An intermediary structure has the ladder geometry and is formed by two strands. While all these planar geometries are more favored energetically than the linear chain, the binding becomes even stronger in nonplanar geometries. We found that by going from bulk to a chain the character of bonding changes and acquires directionality. The conductance of zigzag and linear chains is $4 e^{2} / h$ under ideal ballistic conditions.
\end{abstract}

DOI: 10.1103/PhysRevB.64.195420

PACS number(s): 68.65. $-\mathrm{k}, 73.63 .-\mathrm{b}, 61.46 .+\mathrm{w}, 73.90 .+\mathrm{f}$

\section{INTRODUCTION}

The fabrication of the stable gold monoatomic chains suspended between two gold electrodes is one of the milestones in nanoscience. ${ }^{1,2}$ Issues brought about by this achievement are yet to be resolved: Stable chains were obtained by stretching gold nanowires; no other metal, such as $\mathrm{Al}, \mathrm{Cu}$, has been observed to form a stable monoatomic chain yet. The monoatomic chain, being an ultimate one-dimensional (1D) structure, has been a testing ground for the theories and concepts developed earlier for three-dimensional (3D) systems. For example, it is of fundamental importance to know the atomic structure in a truly 1D nanowire and how the mechanical and electronic properties change in the lower dimensionality.

The density functional theory has been successful in predicting electronic and mechanical properties of bulk metals, where each atom has 8-12 nearest neighbors depending on the crystal structure. While many neighbors in a 3D structure is a signature of the formation of metallic bonds, it is not obvious whether the "metallic" bond picture will be maintained in a monoatomic chain. In fact, for a monoatomic linear chain with one electron per atom, the dimerized state is more stable with a Peierls gap at the zone edge. The situation is expected to be more complex for the chain of aluminum atoms having $3 s^{2} 3 p^{1}$ valency.

The interest in metal nanowires is heightened by the observation of quantized behavior of electrical conductance at room temperature through connective necks stretching between two electrodes. ${ }^{3-7}$ Studies attempting to simulate the process of stretching by using classical molecular dynamics have shown novel atomic and mechanical properties. ${ }^{5,8-14}$ In particular, it was found ${ }^{10}$ that the $2 \mathrm{D}$ hexagonal or square lattice structure of atomic planes perpendicular to the axis changes to the pentagons and later to equilateral triangles when the wire is thinned down to the radius of 5-10 $\AA$. Upon further thinning, strands (or bundles of finite atomic chains), ${ }^{10}$ and eventually a monoatomic chain forms at the narrowest section of the nanowire. ${ }^{10-12}$ Recently, the stability of suspended gold chains and their atomic structures have been studied extensively. ${ }^{15-21}$
The first-principles calculations by Portal et al. ${ }^{15}$ showed that infinite, as well as finite gold atomic chains between two gold electrodes favor the planar zigzag geometry at a bond angle $\alpha=131^{\circ}$. The homogenization of the charge with a depletion in the interatomic region ruled out the formation of a directional chemical bond. On the other hand, the firstprinciples calculations by Häkkinen et al. ${ }^{19,20}$ for a finite gold chain between two gold electrodes favored the dimerized structure. In contrast to the conclusion drawn by Portal et al. ${ }^{15}$ Häkkinen et al. ${ }^{20}$ attributed the stability of the suspended gold chain to the directional local bonding with $s p d$ hybridization. Apparently, the stability of a finite chain depends on the strain and the atomic configuration where the chain is connected to the electrodes. In a more recent comparative study ${ }^{16} \mathrm{Au}, \mathrm{Cu}, \mathrm{Ca}$, and $\mathrm{K}$ infinite chains were found to form planar zigzag structures with equilateral triangular geometry; only Au chain has a second zigzag structure with a wide bond angle $\alpha=131^{\circ}$. Note that these atoms can be considered similar because of their s-type outermostvalence orbitals. Aluminum with $3 s-$, and $3 p$-valence orbitals is different from $\mathrm{Au}, \mathrm{Cu}, \mathrm{Ca}$, and $\mathrm{K}$. Therefore, $\mathrm{Al}$ is an important element for understanding the formation and stability of ultimate 1D atomic chains.

This paper presents a systematic, first-principles analysis of the binding, atomic and electronic structure of very thin $\mathrm{Al}$ chains. The objective is to reveal periodic linear, planar, and nonplanar geometries forming stable structures. An emphasis is placed on the planar structures forming zigzag chains. Some of the Al chain structures are compared with the corresponding structures of $\mathrm{Au}$ chains. It is found that by going from bulk $\mathrm{Al}$ to a chain structure the character of bonding changes and acquires directionality. The higher the coordination of individual atoms, the stronger is the binding energy. It is hoped that the present analysis will contribute to the understanding of atomic structure and related physical properties (such as electrical and thermal conductance, elascticity etc.) of infinite and finite atomic chains.

\section{METHOD}

First-principles calculations were carried out within the density-functional theory. $\mathrm{Al}$ and $\mathrm{Au}$ chains are treated 
within the supercell geometry. To minimize the interchain interaction the distance between the chains is taken to be $20 \AA$. The wave functions are expressed by plane waves with the cutoff energy, $|\mathbf{k}+\mathbf{G}|^{2} \leqslant 275 \mathrm{eV}$. The Brillouin-zone (BZ) integration is performed within Monkhorst-Pack scheme $^{22}$ using $(1 \times 1 \times 40) \mathbf{k}$ points. The convergence with respect to the energy cutoff and number of $\mathbf{k}$ points were tested. Ionic potentials are represented by ultrasoft Vanderbilt-type pseudopotentials ${ }^{23}$ and results are obtained by generalized gradient approximation ${ }^{24}$ (GGA) for fully relaxed atomic structures. Preconditioned conjugate gradient method is used for wave function optimization. Since ionic relaxations are carried out by the conjugate gradient method, the optimized (fully relaxed) structures obtained in this study are stable structures. In certain cases the stability of a structure is tested by calculating the total energy while the atoms are displaced in special directions. Numerical calculations are performed by using VASP code. ${ }^{25}$ The $z$ axis is taken along the chain axis, and $y$ axis ( $x$ axis) is perpendicular to (in) the plane of zigzag structure.

\section{RESULTS}

\section{A. Optimized structures and cohesive energies}

The variation of the total energy $E_{T}$, of the atomic $\mathrm{Al}$ chain calculated for the fully relaxed linear, planar (zigzag and ladder), and nonplanar (cross) structures is shown in Fig. 1. The geometries of these structures and their relevant structural parameters are shown by insets. Since the total energies are given relative to the energy of the free $\mathrm{Al}$ atom, the cohesive energy $E_{C}=-E_{T}$. The zigzag geometry displays two minima; one occurs at $s=1.26 \AA$ and has cohesive energy $E_{C}=2.65 \mathrm{eV} /$ atom; other has shallow minimum and occurs at $s=2.37 \AA$ with cohesive energy $E_{C}$ $=1.92 \mathrm{eV} /$ atom. The high cohesive energy zigzag structure (specified as $T$ ) having the bond length $d=2.51 \AA$, and the bond angle $\alpha \sim 60^{\circ}$ forms equilateral triangles. This geometry allows for four nearest neighbors, which is less than the six nearest neighbors occurring in the $\mathrm{Al}(111)$ atomic plane and 12 nearest neighbors in the close-packed bulk metal. The equilateral triangular geometry can also be viewed as if two parallel linear chains with an interchain distance of $2.17 \AA$ are displaced by 5 along the chain axis ( $z$ direction). This is reminiscent of the hollow site registry of $2 \mathrm{D}$ atomic planes that usually increases the cohesive energy.

The low-cohesive-energy zigzag structure (specified as $W$ ) has $d=2.53 \AA$ and wide bond angle $\alpha \sim 139^{\circ}$, and allows for only two nearest neighbors with bonds slightly larger than those of the $T$ structure. We also found that the cohesive energy decreases if an $\mathrm{Al}$ atom is displaced perpendicular to the zigzag plane. Therefore, both zigzag structures are planar. The minimum energy of the linear structure ( $\alpha$ $=180^{\circ}$ and denoted as $L$ ) has relatively short bond length, $d=s=2.41 \AA$. It is $\sim 0.05 \mathrm{eV}$ above the minimum energy of the $W$ structure and has cohesive energy $E_{C}=1.87 \mathrm{eV} /$ atom.

Two linear chains can form a ladder structure that allows for three nearest neighbors with $\alpha=90^{\circ}$ and $E_{C}$ $\sim 2.4 \mathrm{eV} /$ atom intermediate to the $T$, and $W$ structures. The

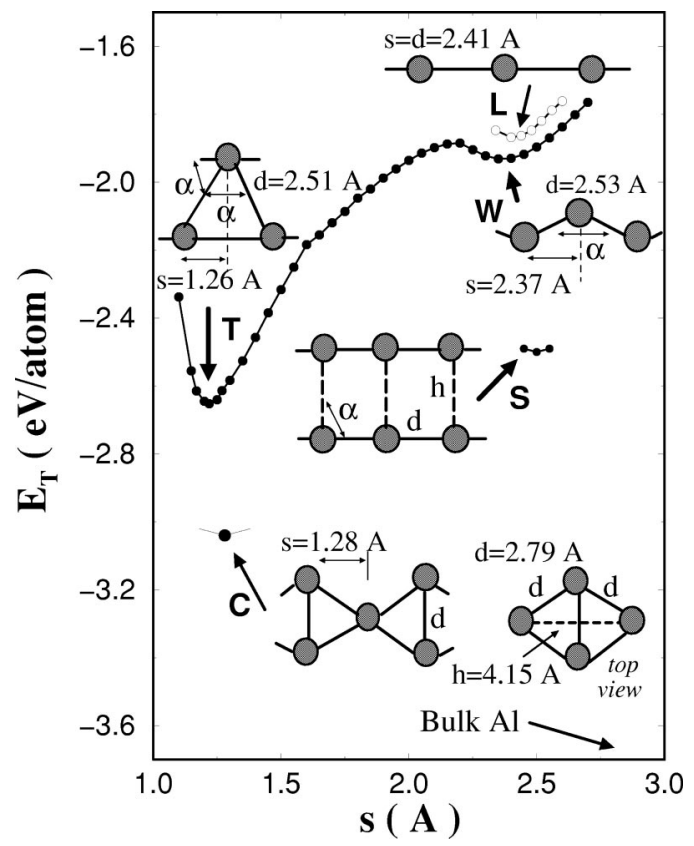

FIG. 1. The calculated total energy $E_{T}$ (and also cohesive energy, $E_{C}$ ) of an infinite $\mathrm{Al}$ chain with linear, planar (zigzag and ladder), and nonplanar (cross) strucures. Energies are given relative to the energy of a free $\mathrm{Al}$ atom. The calculated energy of bulk $\mathrm{Al}$ is indicated by arrow. Relevant structural parameters, bond length $d$, bond angle $\alpha, s=$ and $h$ are shown by inset for nonplanar cross $C$, high energy (or equilateral triangular) $T$, low-energy zigzag $W$, ladder (or strands) $S$ and linear $(L)$ geometries. The zigzag structure is in the $x z$ plane. The short and long dumbbells of the $C$ structure are along $x$ axis and $y$ axis, respectively. For values of energies and structural parameters see Table I.

cohesive energy is further increased to $2.5 \mathrm{eV} /$ atom when the separation between chains is sligtly increasesd. This way two strands (specified as $S$ structure) form, which are held in place by the uniaxial stress between two electrodes. ${ }^{1,10}$

Nonplanar cross structure (specified as $C$ structure) has four atoms that form two perpendicular dumbbells ( $A$ and $B$ ) in the unit cell. The lengths of these dumbells are different $(A: 2.8 \AA$ and $B: 4.15 \AA)$ and the chain is made by the $A B A B A \ldots$ sequence of these dumbbells. $\mathrm{Al}$ atoms in $A$ has five nonplanar bonds, and those in $B$ have four bonds of $\sim 2.8 \AA$. The cohesive energy of this structure is calculated to be $3.04 \mathrm{eV} /$ atom. Since the atoms of the $A$ dumbbells are bound to the nearest five atoms forming equilateral triangles in different planes, and those of the $B$ dumbbels have four nonplanar bonds, this cohesive energy is highest among the 1D structures described in Fig. 1. The $C$ structure was revealed first in the extensive analysis of Gülseren et al. by using empirical glue potential. ${ }^{13}$ The overall features of the stable $C$ structure determined by these calculations are confirmed here, but the structural parameters are more accurately determined by the present first principle calculations.

It is worth noting that the $\mathrm{Au}$ atomic chain also forms two different zigzag structures similar to those of $\mathrm{Al}$; but $\mathrm{Cu}, \mathrm{Ca}$ and $\mathrm{K}$ do not. ${ }^{16}$ Our calculated values for $s, d, E_{C}$ are respectively, $1.36 \AA, 2.71 \AA, 2.23 \mathrm{eV} /$ atom for the $T$ structure; 
TABLE I. Calculated lattice parameters and cohesive energies of $1 \mathrm{D}$ and bulk structures for $\mathrm{Al}$ and $\mathrm{Au}$. The geometric parameters of the structures $(s, d, h$, etc) are explained in Fig. 1.

\begin{tabular}{lcccc}
\hline \hline Structure & $s(\AA)$ & $d(\AA)$ & $\alpha$ & $E_{c}(\mathrm{eV} /$ atom $)$ \\
\hline$L$ & 2.41 & 2.41 & $180^{\circ}$ & 1.87 \\
\hline$W$ & 2.37 & 2.53 & $139^{\circ}$ & 1.92 \\
\hline$S$ & $h=2.68$ & 2.50 & $180^{\circ} \& 90^{\circ}$ & 2.50 \\
\hline$T$ & 1.26 & 2.51 & $60^{\circ}$ & 2.65 \\
\hline$C$ & 1.28 & 2.79 & $60^{\circ} \& 104^{\circ}$ & 3.04 \\
\hline Bulk & & 2.86 & $60^{\circ} \& 90^{\circ}$ & 3.67 \\
\hline$L$ & 2.59 & 2.59 & $180^{\circ}$ & 1.68 \\
\hline$W$ & 2.33 & 2.56 & $131^{\circ}$ & 1.90 \\
\hline$T$ & 1.36 & 2.71 & $60^{\circ}$ & 2.23 \\
\hline Bulk & & 2.96 & $60^{\circ} \& 90^{\circ}$ & 3.20 \\
\hline \hline
\end{tabular}

$2.33 \AA, 2.56 \AA, 1.90 \mathrm{eV} /$ atom for the $W$ structure; $2.59 \AA$, $2.59 \AA, 1.68 \mathrm{eV} /$ atom for the $L$ structure of $\mathrm{Au}$ chain. The nearest neighbor distance of the $T$ structure of the Au chain is reduced by only $\sim 6 \%$ from that of the bulk. Is this puzzling similarity of $1 \mathrm{D}$ atomic structures of $\mathrm{Al}$ and $\mathrm{Au}$ (despite their dissimilar valencies) only a coincidence? We now address this issue.

We calculated the cohesive energy of bulk $\mathrm{Al}(\mathrm{Au}), E_{C}$ $=3.67(3.20) \mathrm{eV} /$ atom at the nearest neighbor distance $d$ $=2.86(2.96) \AA$ [or lattice parameter $a=4.04$ (4.18) $]^{26,27}$ The energetics of $1 \mathrm{D}$ and bulk structures are compared in Table I. Simple arguments based on the counting of nearest neighbor couplings would suggest a relatively small cohesive energy, e.g., $\sim 1.3 \mathrm{eV}$ for the $T$ structure. On the contrary, 1D structures studied here have cohesive energies higher than one can estimate by comparing their coordination numbers with that of bulk. Apparently, the bonds in 1D structures become stronger. In fact, it was found previously that the linear $\mathrm{Al}$ chain has a Young's modulus stronger than bulk. ${ }^{10}$ Recent scanning tunneling microscope studies revealed that the bond strength of the Au nanowire is about twice that of a bulk metallic bond. ${ }^{28}$

\section{B. Charge-density analysis}

Figure 2 shows the charge-density contour plots of bulk, $L, W$, and $T$ structures. In contrast to uniform metallic charge density of bulk, the bonding acquires directionality in $1 \mathrm{D}$ structures of Al. For the $L$ structure the charge is accumulated between atoms forming a directional bond, and is mainly due to the $\sigma$ states (formed by $3 s+3 p_{z}$ orbitals) and partly due to $\pi$ states (formed by $3 p_{x}$ and $3 p_{y}$ orbitals perpendicular to the chain axis). The calculated charge distribu-
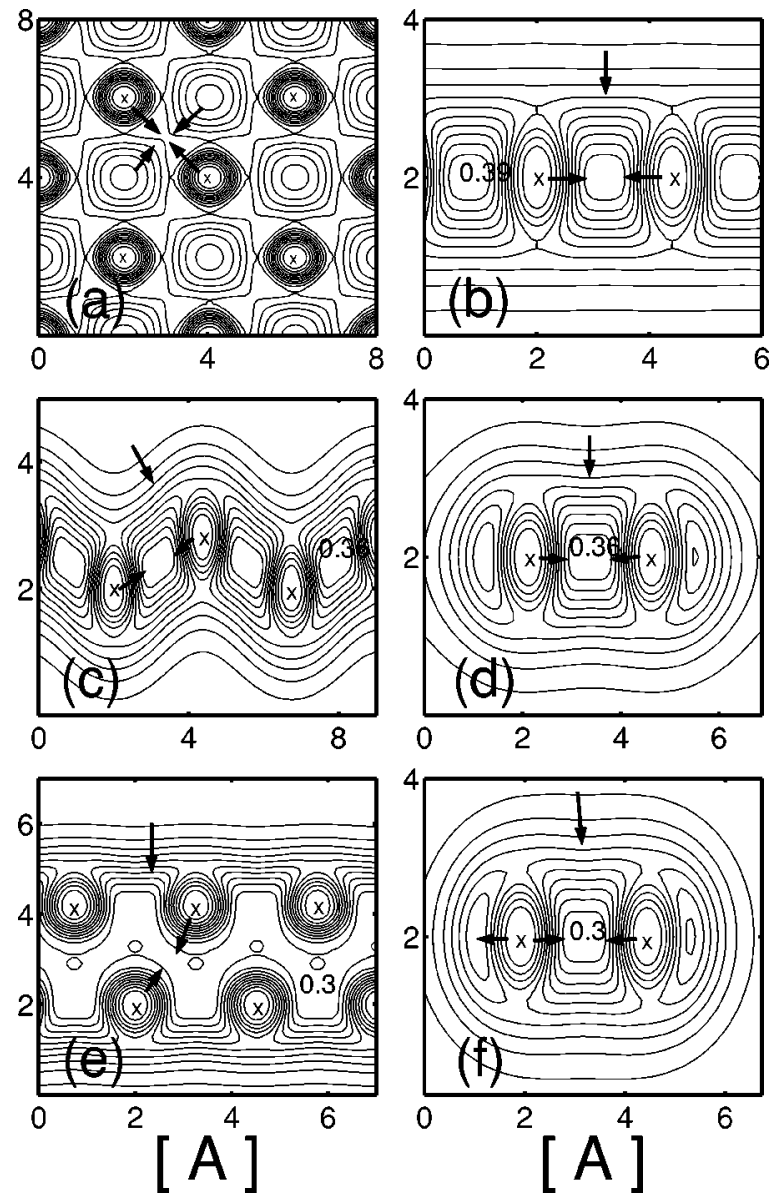

FIG. 2. Charge-density counterplots of 1D and 3D Al structures: (a) Bulk; (b) Linear geometry; (c) $W$ geometry, on the plane of the zigzag structure (i.e., $x z$ plane); (d) $W$ geometry, on the plane passing through the $\mathrm{Al}-\mathrm{Al}$ bond and perpendicular to the plane of the zigzag structure; (e) $T$ geometry, on the plane of the zigzag structure; (f) $T$ geometry, on the plane passing through the bond and perpendicular to the plane of the zigzag structure. Increasing direction of the charge density is indicated by arrows. Numerals show the highest-contour values. Atomic positions are indicated by $\mathrm{x}$.

tion suggests that directional "covalent" bonds are responsible for the bonding. This situation is maintained in the zigzag $W$ structure except for a slight distortion of the bond charge. Actually, the $W$ structure with wide bond angle is not dramatically different from the $L$ structure. In the $T$ structure that forms equilateral triangles, the charge density is apparently different from that of the $W$ structure. We see a continuous (connected) region of high charge density between double atomic chains. However, this is nothing but the overlap of charges of four bonds emerging from each chain atom, and is confirmed by the contour plot of an individual bond charge in a plane perpendicular to the zigzag $x z$ plane and passing through an $\mathrm{Al}-\mathrm{Al}$ bond. We also notice that the charge becomes slightly delocalized by going from $L$ to $T$ structure. These charge distributions of the $\mathrm{Al}-\mathrm{Al}$ bond described above is different from the corresponding charge distribution of Au zigzag structures shown in Fig. 3. Clearly, there are no directional bonds in the $\mathrm{Au}$ chain; valence charge is delocalized. This finding is in confirmity with the 

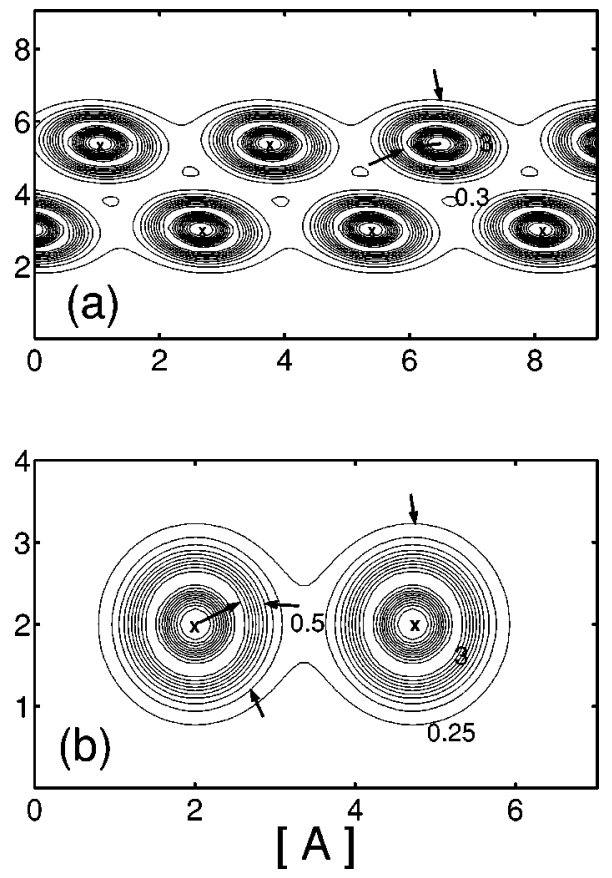

FIG. 3. Charge-density counterplots of the Au chain. (a) $T$ structure; (b) $T$ structure, on the plane passing through the bond and perpendicular to the zigzag plane.

results of Portal et al. ${ }^{15,16}$ on the infinite Au chain. On the other hand, Häkkinen et al. ${ }^{20}$ deduced directional bonding with spd hybridization in finite $\mathrm{Au}$ chains between two $\mathrm{Au}$ electrodes by performing similar type of pseudopotential plane wave calculations.

\section{Electronic structure}

A comparative analysis of the electronic band structure of Al monoatomic chains illustrated in Fig. 4 provides further insight into the stability and character of bonding. The band structure of the $L$ structure is folded for the sake of comparison with the zigzag structures. Two filled $\sigma$ bands arise from the $3 s+3 p_{z}$ valence orbitals and make the bond charge shown in Fig. 2(a). Because of the linear geometry $3 p_{x}$ and $3 p_{y}$ are equivalent and give rise to doubly degenerate $\pi$ band crossing the Fermi level. As pointed out by Peierls, ${ }^{30}$ a one-dimensional metal with a partly filled band will distort away from a regular chain structure to lower its energy. According to the above analysis, a linear chain of uniformly spaced $\mathrm{Al}$ atoms with spacing $s=d$ has a quarter-filled band that crosses the Fermi level at $k_{z}= \pm \pi / 4 d$. A distorted unit cell $4 d$ in length will cause this point to coincide with the edge of the Fermi distribution. The change in the crystal potential due to the $4 d$ distortion will open up a Peierls gap at the reduced zone edge lowering the total energy. In practice, the gain in energy due to such Peierls distortions is rather small even for a $2 d$ dostortion (dimerization) and is likely to be below computational error for the $4 d$ distortion here. Thus, although the linear chain of $\mathrm{Al}$ atoms is unstable, in principle, the effect of such a distortion on cohesive energy is clearly negligible.

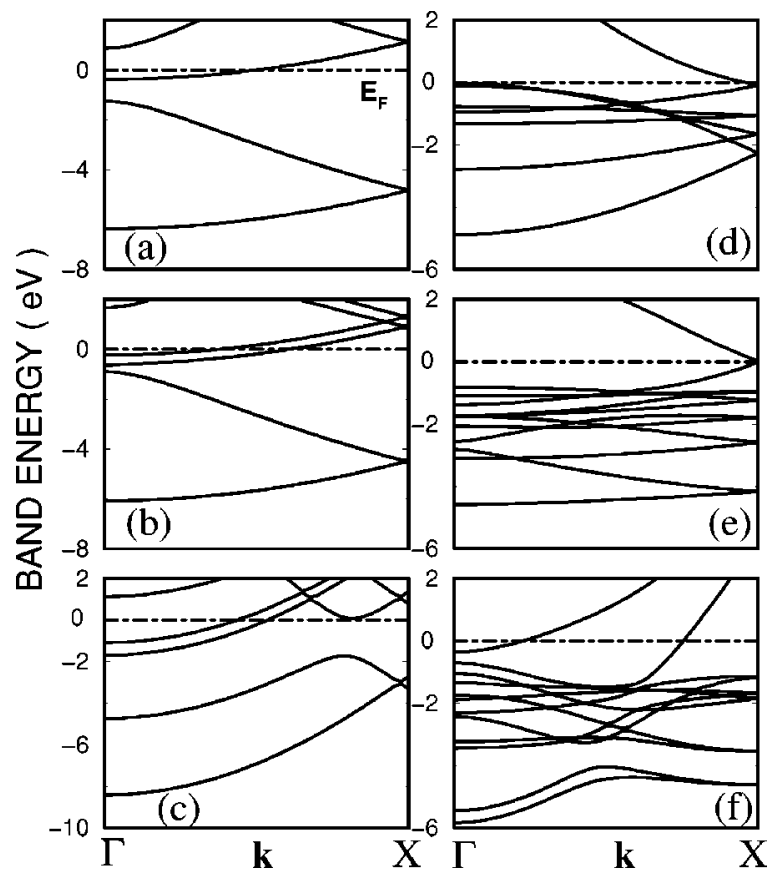

FIG. 4. Energy band structure of $\mathrm{Al}$ and Au chains. (a) Linear $L$ structure; (b) low-energy zigzag, $W$ structure; (c) high-energy zigzag $T$ structure of Al. (d) Linear $L$ structure; (e) $W$ structure; (f) $T$ structure of $\mathrm{Au}$. Bands of $L$ structure is zone folded for the sake of comparison with the zigzag structures. Zero of energy is taken at the Fermi level.

The symmetry between $3 p_{x}$ and $3 p_{y}$ orbitals is broken in the zigzag structure, and hence the $\pi$ band is split. Apart from this band splitting and slight rise of bands, the overall form of the energy band structure is maintained in the $W$ structure. The $W$ structure is, however, more stable than the $L$ structure because of its relatively stronger electronic screening. In the $T$ structure the split $\pi$ bands are lowered, and the form of the $\sigma$ bands undergo a significant change due to the equilateral triangular geometry. Despite slight delocalization of charge, the total energy of the $T$ structure is lower than the $W$ structure. The relative stability originates from the increased number of nearest neighbors. It is noted that the bands in all chain structures can be considered similar as far as Fermi level crossing of the bands is concerned.

In contrast to the Al chains described above, the energy band structure of Au undergoes significant changes in different structures near the Fermi energy. For example, for the linear structure one band crosses the Fermi level near the $X$ point of the BZ, another band at the $\Gamma$ point is very close to the Fermi level. For the $W$ structure, two bands cross at the Fermi level and at the zone boundary with negligible Peierls distortion gap $^{29}$ and the rest of the bands are lowered. The lowering of the state density at the Fermi level stabilizes the zigzag structure relative to the linear structure. ${ }^{15,16}$ In the $T$ structure two bands cross the Fermi level. Inspite of these changes the character of the bonding remains essentially metallic in the chain structures.

\section{DISCUSSION AND CONCLUSIONS}

The analysis of the above results and comparison with $\mathrm{Au}$ chain reveal that the metallic bond of bulk Al changes to a 
directional covalent bond in the 1D monatomic chain. The metallicity is ensured by the $\pi$ states. For that reason, our efforts of calculating the bands of the $\mathrm{Al}$ chains with tightbinding method by using the bulk parameter ${ }^{31}$ have not been too successful. This suggests that the transferability of energy parameters fitted to bulk is not satisfactory for the 1D $(T, W$, and $L)$ structures.

The $T$ structure with two parallel linear chains can be viewd as the $1 \mathrm{D}$ analog of the $3 \mathrm{D}$ close packing. In this respect, the $T$ structure may be considered in a different class and as a precursor of the $2 \mathrm{D}$ hexagonal lattice. Adding one more parallel chain in registry with the quasi 1D $T$ structure, one starts to build the hexagons, where two-thirds of the atoms have four and one-third of the atoms have six nearest neighbors. As a natural extention of these arguments, another intermediary, quasi-1D structure, for example, is a ladder structure that consists of two parallel linear chains forming a row of squares with a lattice constant of $d$ and allowing for three nearest neighbors. This metastable structure is a $1 \mathrm{D}$ analog of the top site registry of $2 \mathrm{D}$ atomic planes. ${ }^{32}$ Our calculations show that the cohesive energy of the ladder structure is increased when the distance between two chains increases, so that the chain turns to two strands. The cohesive energy of the strands is found between the $T$ and $W$ structure. By going from planar to nonplanar geometry the cohesive energy further increases. The present work suggests that the Born-Oppenheimer surface for these quasi-1D structures is rather complex and generally $E_{C}$ increases with increasing coordination number and decreasing bond angle. As clarified in Sec. II, the stable structures correspond to the local minima on the Born-Oppenheimer surface and are expected to be vibrationally stable at least at low temperatures. The 1D $T$ structure found for $\mathrm{Al}, \mathrm{Au}, \mathrm{Cu}, \mathrm{Ca}$, and $\mathrm{K}$ appears to be common to metals, in a way an intermediate structure between a truly $1 \mathrm{D}$ and $2 \mathrm{D}$ structures.

We also note that the linear and zigzag structures of $\mathrm{Al}$ have two bands crossing the Fermi energy. Calculations using the Green's function method ${ }^{33}$ yield one conduction channel for each band of uniform chain crossing the Fermi level, and hence the ballistic conductance of the $T, W$, and $L$ structures $G=2\left(2 e^{2} / h\right)$. This value for the conductance arises from the fact that the channel capacity or the maximum conductance per channel is $2 e^{2} / h$. It is straightforward to motivate the maximum value by appealing to the Heisenberg's uncertainity principle. ${ }^{34}$ Recalling that conductance $G=\Delta I / \Delta V$, and $\Delta I=\Delta Q / \Delta t$, then for a single channel in extreme quantum limit $\Delta Q=e$. One can readily write $G$ $=e^{2} / \Delta E \Delta t$. Now invoking the uncertainity principle, $\Delta E \Delta t \geqslant h$, one finally obtains $G \leqslant 2 e^{2} / h$. Here the factor of 2 is due to spin. The maximum conductance per channel can never be greater than $2 e^{2} / h$. We note that the value of the ballistic conductance for the infinite chain (i.e., $4 e^{2} / h$ ) is at variance with the experimental results ${ }^{35}$ yielding only $G$ $\sim 2 e^{2} / h$ for the finite $\mathrm{Al}$ chain. The discrepancy was explained by the fact that the electronic states are modified due to the finite size of the chain and the atomic configuration where the chain is coupled to the electrodes. ${ }^{36}$

It is important to remark that creation of metallic overlayers on semiconductors is required in chip technology. Thus if one could place these quasi-1D structures on semiconductor surfaces without losing the metallic behavior of the chains, the technology would be considerably enhanced. Unfortunately, when one examines the deposition of monolayers of metals like $\mathrm{Al}, \mathrm{Au}$ and $\mathrm{Ga}$ on $\mathrm{Si}$, the lowest energy configuration turns out to be semiconducting in nature. A metastable state, in which $\mathrm{Al}$ forms a metallic zigzag structure on the $\mathrm{Si}(100)$ surface, has been reported. ${ }^{37}$ It remains to be seen how feasible it is to fabricate such a structure.

In summary, we have found that a zigzag chain of aluminum in triangular configuration is most stable among the planar structures we studied. The structural results for planar geometries are similar to gold but bonding is different. A metastable ladder structure intermediate to distorted linear and triangular structure is also reported. The metallicity has its origin in the $\pi$ bands. The stabilization of these metallic monoatomic chains on semiconductors remain an experimental challenge.

\section{ACKNOWLEDGMENTS}

We thank Dr. Oguz Gülseren for stimulating discussions.
${ }^{1}$ H Ohnishi, Y. Kondo, and K. Takayanagi, Nature (London) 395, 780 (1998).

${ }^{2}$ A.I. Yanson, G.R. Bolligert, H.E. Brom, N. Agraï, and J.M. Ruitenbeek, Nature (London) 395, 783 (1998).

${ }^{3}$ N. Agraït, J.G. Rodrigo, and S. Vieria, Phys. Rev. B 47, 12345 (1993)

${ }^{4}$ J.I. Pascual, J. Méndez, J. Gómez-Herrero, M. Barró, N. Garcia, and Vu Thien Binh, Phys. Rev. Lett. 71, 1852 (1993).

${ }^{5}$ J.I. Pascual, J. Méndez, J. Gómez-Herrero, M. Barró, N. Garcia, U. Landman, W.D. Luedtke, E.N. Bogacheck, and H.-P. Cheng, Science 267, 1793 (1995).

${ }^{6}$ L. Olesen, E. Laegsgaard, I. Stensgaard, F. Besenbacher, J. Schiøtz, P. Soltze, K. W. Jacobsen, and J. K. Nørskov, Phys. Rev. Lett. 72, 2251 (1994).
${ }^{7}$ J.M. Krans, C.J. Müller, I.K. Yanson, Th.C.M. Govaert, R. Hesper, and J.M. van Ruitenbeek, Phys. Rev. B 48, 14721 (1993).

${ }^{8}$ U. Landman, W.D. Luedtke, N.A. Burnham, and R.J. Colton, Science 248, 454 (1990).

${ }^{9}$ A.M. Brotkovsky, A.P. Sutton, and T.N. Todorov, Phys. Rev. B 52, 5335 (1995).

${ }^{10}$ H. Mehrez and S. Ciraci, Phys. Rev. B 56, 12632 (1997).

${ }^{11}$ M.R. Sbrensen, M. Brandbyge, and K.W. Jacobsen, Phys. Rev. B 56, 14956 (1997).

${ }^{12}$ G.M. Finbow, R.M. Lynden-Bell, and I.R. McDonald, Mol. Phys. 92, 705 (1997).

${ }^{13}$ O. Gulseren, F. Ercolesi, and E. Tosatti, Phys. Rev. Lett. 80, 3775 (1998).

${ }^{14}$ E. Tosatti and S. Prestipino, Science 289, 561 (2000); E. Tosatti, 
S. Prestipino, S. Kostlmeier, A. Dal Corso, and F. D. Di Tolla, ibid. 291, 288 (2001).

${ }^{15}$ D.S. Portal, E. Atracho, J. Junquera, P. Odrejón, A. García, and J.M. Soler, Phys. Rev. Lett. 83, 3884 (1999).

${ }^{16}$ D.S. Portal, E. Atracho, J. Junquera, A. García, and J.M. Soler, cond-mat/0010501 (unpublished).

${ }^{17}$ J.A. Torres, E. Tosatti, A.D. Corso, F. Ercolessi, J.J. Kohanoff, F.D. Di Tolla, and J.M. Soler, Surf. Sci. 426, L441 (1999).

${ }^{18}$ M. Okamoto and K. Takayanagi, Phys. Rev. B 60, 7808 (1999).

${ }^{19}$ H. Häkkinen, R.N. Barnett, and U. Landman, J. Phys. Chem. B 103, 8814 (1999).

${ }^{20}$ H. Häkkinen, R.N. Barnett, A.G. Scherbakov, and U. Landman, J. Phys. Chem. B 104, 9063 (2000).

${ }^{21}$ L. De Maria and M. Springborg, Chem. Phys. Lett. 323, 293 (2000).

${ }^{22}$ H.J. Monkhorst and J.D. Pack, Phys. Rev. B 13, 5188 (1976).

${ }^{23}$ D. Vanderbilt, Phys. Rev. B 41, 7892 (1990); G. Kresse and J. Hafner, J. Phys.: Condens. Matter 6, 8245 (1994).

${ }^{24}$ J.P. Perdew and Y. Wang, Phys. Rev. B 46, 6671 (1992).

${ }^{25}$ G. Kresse and J. Hafner, Phys. Rev. B 47, R558 (1993); G. Kresse and J. Furtmüller, ibid. 54, 11169 (1996).

${ }^{26}$ F.D. Tolla, A.D. Corso, J.A. Torres, and E. Tosatti, Surf. Sci. 454-456, 947 (2000). In this paper the conductances of the $L$ and $C$ structures are treated by using the first-principles bandstructures calculations.

${ }^{27}$ The bulk cohesive energies calculated in the present work are in good agreement with earlier calculations using similar scalarrelativistic pseudopotentials. For example, in the local-density approximation (LDA), we obtained for the bulk $\mathrm{Au} d$ $=2.88 \AA, E_{C}=4.4 \mathrm{eV}$. In the GGA, $d=2.96 \AA, E_{C}$
$=3.20 \mathrm{eV}$. The values given in by Portal et al. ${ }^{15}$ are $d$ $=2.91 \AA, \quad E_{C}=4.55 \mathrm{eV}$ for LDA, and $d=2.98 \AA, E_{C}$ $=3.37 \mathrm{eV}$ for GGA. The experimental values are $d=2.87 \AA$, $E_{C}=3.78 \mathrm{eV}$. The present GGA calculations found $d$ $=2.86 \AA$ А $E_{C}=3.67 \mathrm{eV}$ for the bulk Al, and the GGA calculations by Torres et al. ${ }^{17}$ obtained $d=2.79 \AA, E_{C}=3.74 \mathrm{eV}$. The corresponding experimental values for $\mathrm{Al}$ are $d=2.86 \AA$ and $E_{C}=3.39 \mathrm{eV}$. The GGA has a tendency towards underbinding, in particular for the noble metals $\mathrm{Cu}, \mathrm{Ag}, \mathrm{Au}$, while the LDA is overbinding. The GGA outperforms the LDA when it comes to the cohesive energie of Al. It was shown that fully relavistic calculations improve the cohesive energies in the GGA. These features have been extensively discussed. See, for example, P.H.T. Philipsen and E.J. Baerends, Phys. Rev. B 61, 1773 (2000).

${ }^{28}$ G. R. Bolliger, S. R. Bahn, N. Agraï, K. W. Jacobsen, and S. Vieira, (to be published).

${ }^{29}$ I.P. Batra, Phys. Rev. B 42, 9162 (1990).

${ }^{30}$ R.E. Peierls, Quantum Theory of Solid (Oxford University Press, London, 1955).

${ }^{31}$ D.A. Papaconstantopoulos, Handbook of the Band Structure of Elemental Solids (Plenum Press, New York, 1986).

${ }^{32}$ S. Ciraci, A. Baratoff, and I.P. Batra, Phys. Rev. B 42, 7618 (1990); 46, 10411 (1992).

${ }^{33}$ A. Buldum and J.P. Lu, Phys. Rev. B 63, R161403 (2001).

${ }^{34}$ I.P. Batra, Surf. Sci. 395, 43 (1998).

${ }^{35}$ E. Scheer, N. Agrait, J.C. Cuevas, A.L. Yeyali, B. Ludoph, A.M. Rodero, G.R. Bollinger, J.M. van Ruitenbeck, and C. Urbina, Nature (London) 394, 154 (1998).

${ }^{36}$ H. Mehrez, S. Ciraci, A. Buldum, and I.P. Batra, Phys. Rev. B 55, R1981 (1997).

${ }^{37}$ I.P. Batra, Phys. Rev. Lett. 63, 1704 (1989). 